\title{
Multiaxial Fatigue Behavior of High-Density Polyethylene (HDPE) Including Notch Effect: Experiments and Modeling
}

\author{
Mohammadreza Amjadi, Ali Fatemi
}

Mechanical Engineering Department, The University of Memphis, Memphis, TN, USA

\begin{abstract}
High-Density Polyethylene (HDPE) is used in many industries with many applications from automotive industry to biomedical implants. It can be manufactured using different processing techniques including compression molding, injection molding, and blow molding. Multiaxial loading and non-proportionality between different loading sources are inevitable in many applications. It is shown that the common multiaxial fatigue criteria such as von Mises equivalent stress are not able to correlate the multiaxial fatigue data. In this study, multiaxial fatigue behavior of neat HDPE is investigated using hollow tubular specimens through experimental fatigue tests. Axial, torsion, and combined in phase and out-of-phase axialtorsion fatigue tests were conducted. Stress concentration effect on multiaxial fatigue behavior was also studied. Experimental results and analytical models used to account for the aforementioned effects are presented and discussed in this paper.
\end{abstract}

\section{Introduction}

Application of polymers in different industrial applications has been increasing in recent decades, therefore, assessment of their mechanical behaviour is important in many design situations. Plastic components must function during service life without failure under cyclic loading. Hence, they need to be designed based on cyclic service loads considering different effects including multiaxial stress state and stress concentrations.

High-Density Polyethylene (HDPE) is the most used of the three polyethylene grades for a wide range of applications. Global demand for HDPE resins has been increasing, going from 11.9 million tons in 1990 to 43.9 million tons in 2017 with annual growth of $3.3 \%$ [1]. The physical properties exhibited by polyethylene products are governed by the relative proportions of the crystalline and non-crystalline phases. Molecular constitution and microstructural aspects like the degree of crystallinity, crystal size, and crystal orientation are essential to determine mechanical response of polyethylene. Higher molecular weight leads to higher strength due to the low capability of sliding molecules over each other. On the other hand, increasing molecular weight and branching reduce crystallinity.

Similar to metallic materials, under cyclic loading most polymers will fracture at stress levels much lower than they can withstand under static loading. Despite the fact that the fatigue failure phenomenon in metals has been investigating since the 18th century, research 
on polymer fatigue is much more recent [2]. Most of the research conducted on fatigue behavior of semicrystalline thermoplastics is focused on uniaxial (tension-tension or tensioncompression) loading condition. However, few studies evaluated the multiaxial stress state effect on fatigue behavior of thermoplastics and their short fiber composites.

It has been shown that microstructural parameters such as crystallinity, molecular orientation, and molecular distribution affect mechanical properties of thermoplastics [3-8]. The degree of crystallinity has more effect on mechanical properties than molecular weight for HDPE [9, 10]. Crystallinity and secondary bond strength control the stiffness, while intrachain, inter-chain, secondary bonding, and crystallinity contribute to the strength of thermoplastics. Crystalline regions increase strength, while restricting the deformation in the amorphous phase due to the extension of molecules in the non-crystalline areas. Processing conditions can influence the crystal above microstructure parameters and consequently mechanical properties [11]. For example, temperature gradient in melted resin during the manufacturing process affects crystallinity, i.e. higher mold temperature leads to lower crystallization rate [10].

Chen et al. [12] investigated cyclic deformation and self-heating of Ultra-High Molecular Weight Polyethylene (UHMWPE). Under cyclic loading, the behavior was observed as a cyclic stabilized material, except for very high loading rate where the temperature rise caused thermal softening (strain rate of $0.1 \mathrm{~s}^{-1}$ ). Moreover, mean stress relaxation occurred in the asymmetrical strain-controlled cyclic tests of the UHMWPE, but the applied mean strain hardly influenced the temperature rise on the specimen surface. A significant amount of ratcheting was observed during the asymmetrical stress-controlled cyclic loading which depended on the applied mean stress and stress amplitude.

Berrheli et al. [13] studied multiaxial fatigue behavior of HDPE at room temperature using stress-controlled fatigue tests in axial (tension-compression) and torsion at stress ratios of $R=0$ and $R=-1$. There was an influence of loading mode on monotonic behavior under tension, compression, and torsion loading. However, the von Mises equivalent stress at the onset of instability (necking) did not appear to be very dependent on the loading mode. Significant cyclic softening was observed at $R=0$ for either tension or torsion loading. At a given $R$ ratio, the torsion and axial fatigue data successfully came together by the von Mises equivalent stress. It was concluded that the fatigue life behavior of HDPE strongly depends on shear processes. In order to capture the effect of mean stress, an equivalent stress was proposed. This equivalent stress was the summation of maximum second invariant tensor and mean second invariant tensor.

This paper presents experimental data for multiaxial fatigue behavior of HDPE, including notch effect. Crack initiation orientations were identified for both un-notched and notched specimens under torsion and combined in-phase axial-torsion loading. The commonly used von Mises criterion as well as the critical plane approach are used to correlate multiaxial fatigue lives under axial, torsion, in-phase axial-torsion, and out-of-phase axial-torsion loading conditions.

\section{Material and Methods}

Tubular specimens with $2 \mathrm{~mm}$ wall thickness and internal diameter of $23 \mathrm{~mm}$ in the gage section were machined from extruded HDPE solid bars as shown in Fig. 1. Finite element analysis (FEA) was performed under axial load to evaluate the stress distribution. As shown in Fig. 1, there is nearly uniform stress distribution at the middle of gage section. About $5 \mathrm{~mm}$ 
length at the centre of the specimen along longitudinal direction is under $97 \%$ of nominal stress. This allows for the observation of cracking behavior in a nearly uniform stress field. This geometry was also chosen to minimize temperature rise due to self-heating. For notch specimen tests, a transverse circular hole with $2 \mathrm{~mm}$ diameter was drilled in the middle of the gage section (at minimum thickness) and nominal stresses with respect to gross cross sectional area were used in stress calculations. Stress concentration factors for notched specimens in axial and torsion loadings were computed as $K_{t a}=2.94$ and $K_{t t}=1.75$, respectively.

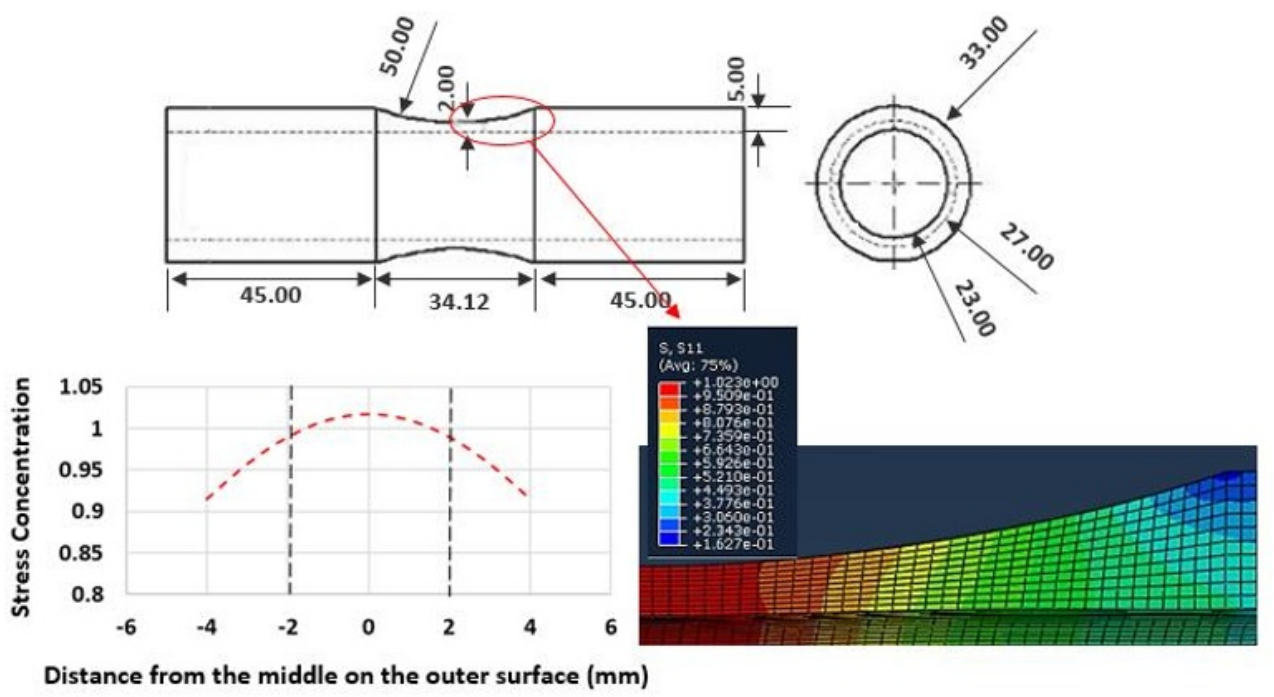

Fig. 1. Specimen geometry used for multiaxial fatigue testing. There is nearly uniform stress distribution zone ( $5 \mathrm{~mm}$ length) in the middle of gage section based on finite element analysis for axial load. A transverse circular hole was drilled in the middle of the gage section for notched specimens (dimensions are in $\mathrm{mm}$ ).

The common mechanical and physical properties of HDPE material are reported in Table. 1.

Table 1. Mechanical and physical properties of HDPE.

\begin{tabular}{c|c|c} 
Property & Value & Unit \\
\hline Density, $\rho$ & 0.96 & $\mathrm{~g} / \mathrm{cm}^{3}$ \\
Elastic Modulus, $E$ at $23^{\circ} \mathrm{C}$ & 1500 & $\mathrm{MPa}$ \\
Ultimate Tensile Strength, $S_{u}$ at $23^{\circ} \mathrm{C}$ & 27 & $\mathrm{MPa}$ \\
Percent Elongation @ Ultimate Tensile Strength $23^{\circ} \mathrm{C}$ & 10 & $\%$ \\
Glass Transition Temperature, $T_{g}$ & -110 & ${ }^{\circ} \mathrm{C}$ \\
Melting Temperature, $T_{m}$ & 140 & ${ }^{\circ} \mathrm{C}$
\end{tabular}

All multiaxial fatigue tests were performed at room temperature on a servo-hydraulic axial-torsion testing machine. Fully reversed $(R=-1)$ load-controlled tests were conducted under axial, torsion, in-phase axial-torsion, and $90^{\circ}$ out-of-phase axial-torsion loading conditions using a sinusoidal wave shape. Tests were conducted at a frequency range of 0.1 to $1.4 \mathrm{~Hz}$. An infrared camera was used to monitor the temperature at the middle of the specimen and a fan was used to cool down the specimen and keep the temperature rise below $5^{\circ} \mathrm{C}$ during cyclic loading. 
Stress calculations were done according to ASTM stranded for strain-controlled axialtorsional fatigue testing with thin walled tubular specimens [14]. For combined axial-torsion tests stress ratio $(\tau / \sigma)$ of $\sqrt{3} / 3$ was chosen.

\section{Result and Discussion}

\subsection{Damage Mechanism}

Microcracks may be formed in the polymeric materials during static or fatigue cyclic loadings. These micro cracks can propagate as the structure is exposed to variable environmental or mechanical loading. HDPE is a semicrystalline polymer, which composed of amorphous and crystalline phases. There are always defects and voids in the HDPE material depending on manufacturing conditions such as cooling rate, thickness of the part, which can act as the critical stress concentration locations to initiate cracks. The fatigue failure of polymers begins by the initiation of a crack in a surface craze or at a surface defect or at a stress concentration point $[15,16]$.

Initial damage takes place by deformation of the spherulites, followed by the transformation of spherulites into fibrils which are themselves subsequently elongated [15]. Fatigue damage is believed to occur initially by inter- and trans-spherulitic crazing however, the cyclic compression which occurs in the near crack-tip regions crushes the spherulites and elongates them in the direction of crack growth [15].

Plastic deformation of HDPE is a complex phenomenon and it is not only based on crazing and shear but it includes the deformation and interaction of both phases together. Unlike crystalline lamellae, amorphous phase has much lower stiffness, hence it deforms easily at smaller stress. The crystalline lamella acts as a constraint against the deformation in amorphous phase. The deformation of amorphous phase results in the interlamellar shear, rotation of lamellar stacks or separation of lamellae which depends on the local stress field [17]. In semi-crystalline polymers deformed at temperatures above $T_{g}$ cavitation occurs and microcavities (microvoids) form inside the bulk of a polymer deformed in solid state.

Generally, cracks do not form randomly under fatigue loading and there is typically a preferred orientation for crack initiation orientation and propagation, depending on microstructural characteristics and multiaxial stress state in the material. Knowing the damage mechanism is key to selection of proper predictive fatigue model. Therefore, a critical plane approach for multiaxial fatigue behavior of HDPE consistent with its failure mechanism is desirable. Crack initiation locations in both un-notched and notched specimens under torsional and in-phase axial-torsion loading were examined under optical microscope to determine crack initiation orientation. Once cracks nucleate, their growth can be divided in two stages, stage I associated with micro-crack growth along maximum shear planes, and stage II related to crack growth along maximum tensile stress plane. Micro-crack growth life of ductile-behaving materials is typically dominated by stage I growth [18].

Crack orientation resulting from cyclic loading for un-notched and notched specimens under torsion and axial-torsion loadings are shown in Fig. 2. For un-notched specimens under torsion, cracks imitated and propagated along maximum shear plane (Fig. 2(a)). However, cracks initiated on maximum shear plane but grew in maximum principal stress for unnotched test under in-phase axial-torsion (Fig 2(b, c)). The difference in crack growth orientation between torsion and in-phase axial-torsion loadings may be explained by the normal stress effect which is higher in in-phase than torsion loading. Cracks initiated on maximum shear plane and grew on maximum principal plane for notched specimens under either torsion or in-phase loading, as shown in Fig. 2(d, e). 


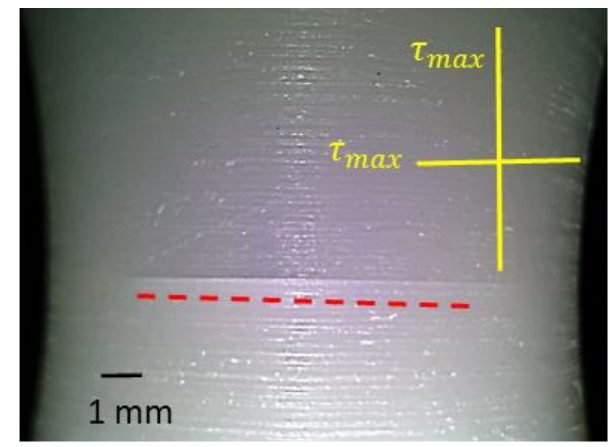

a

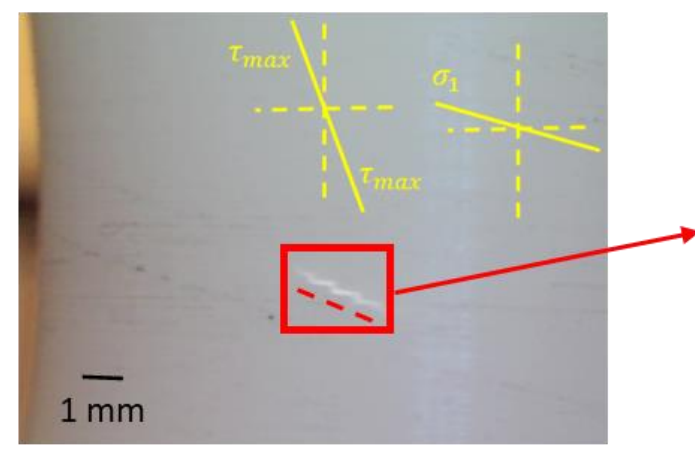

b

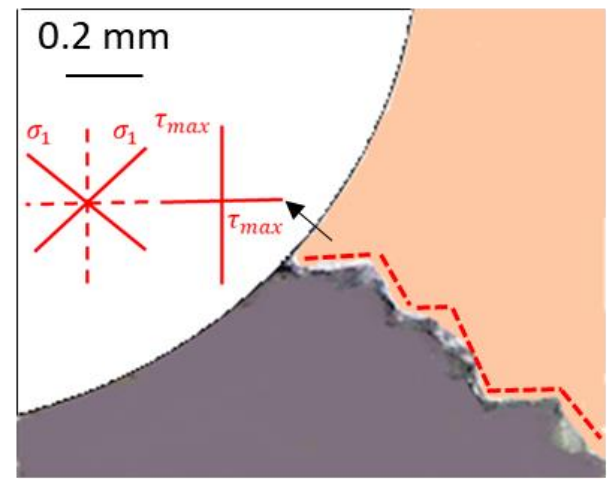

d

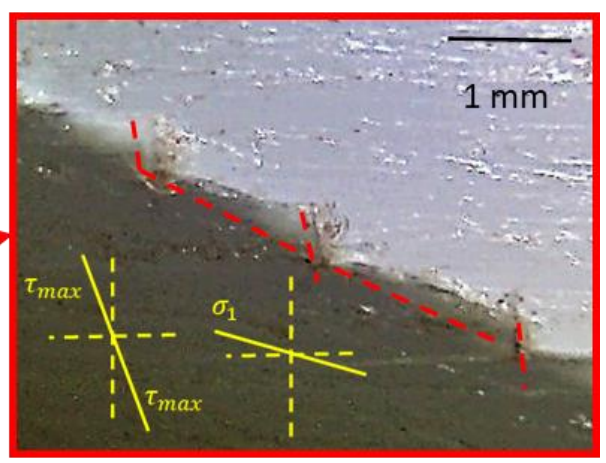

C

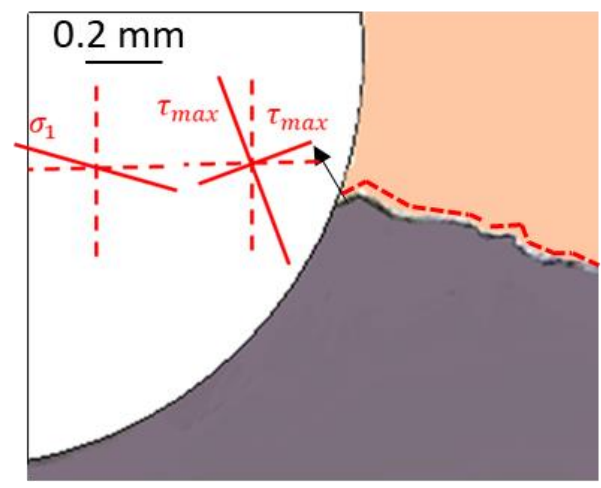

e

Fig. 2. Crack initiation and growth orientations for un-notched specimens under torsion (a), in-phase axial-torsion (b, c), notched tests under torsion (d), and in-phase axial-torsion loading (e).

\subsection{Un-Notched Multiaxial Fatigue Test Results and Data Correlations}

The most common used criterion in multiaxial stress state under static load is equivalent von Mises stress, which is the shear stress on the octahedral plane with respect to maximum principal stress axes. There are three main methods in the literature for multiaxial fatigue analysis. Stress-based (stress invariant), strain-based, and energy models have been used to describe multiaxial fatigue behavior. Fatigue analysis and life prediction using the concept of critical plane approaches is very effective since the critical plane concept is based on the 
failure mode or damage mechanism to initiate cracks in material. The Fatemi-Socie (FS) critical plane damage parameter is a common multiaxial fatigue parameter based on the maximum shear strain amplitude and the maximum normal stress on the maximum shear strain range plane, given by:

$$
D=\frac{\Delta \gamma_{\max }}{2}\left(1+k \frac{\sigma_{n, \max }}{S_{y}}\right)
$$

where $\Delta \gamma_{\max }$ is maximum shear strain range, $\sigma_{n, \max }$ is the maximum normal stress acting on the maximum shear strain plane over the cycle and $S_{y}$ is the yield strength. Gates and Fatemi [19] suggested replacement of yield strength in the original form [20] with $G \Delta \gamma$ [21]. A stress version of this parameter with can be written as:

$$
D=\tau_{a}\left(1+k \frac{\sigma_{n, \max }}{\tau_{a}}\right)=A N_{f}^{b}
$$

where $\tau_{a}$ is the shear stress amplitude, $\sigma_{n, \max }$ is the maximum normal stress, and $k$ is a material constant. Under cyclic multiaxial loading, this damage parameter is calculated on different planes and the plane with maximum amount of damage is defined as the crack initiation plane.

Multiaxial fatigue data correlations for HDPE are shown in Fig. 3. Data correlations based on the commonly used equivalent stress criterion are shown in Fig. 3(a). Similar to many ductile behaving metallic materials, von Mises criterion can bring the multiaxial data for axial, torsion and in-phase axial-torsion loading together, however this is not the case for the out-of-phase loading data. As can be seen in Fig. 3(a), there is a significant reducing effect on fatigue life due to load non-proportionality. The stress-based critical plane-based damage parameter (equation 2) on the other hand correlates all the data well, as can be seen from Fig. 3(b). This shear based critical plane-based damage parameter is consistent with the damage mechanism of HDPE as discussed in section 3.1.

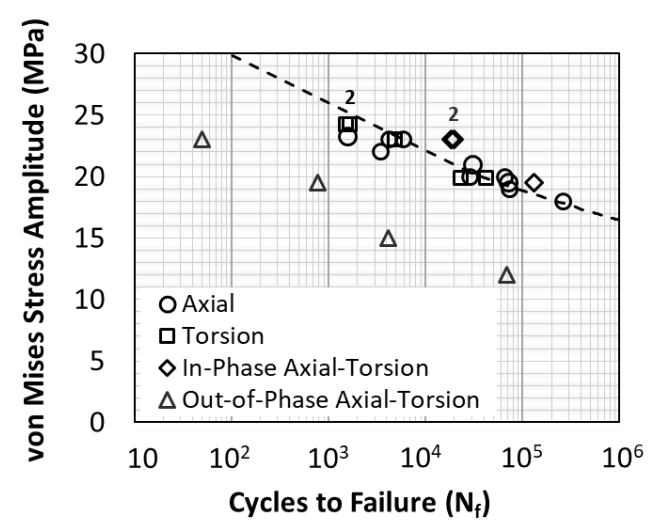

a

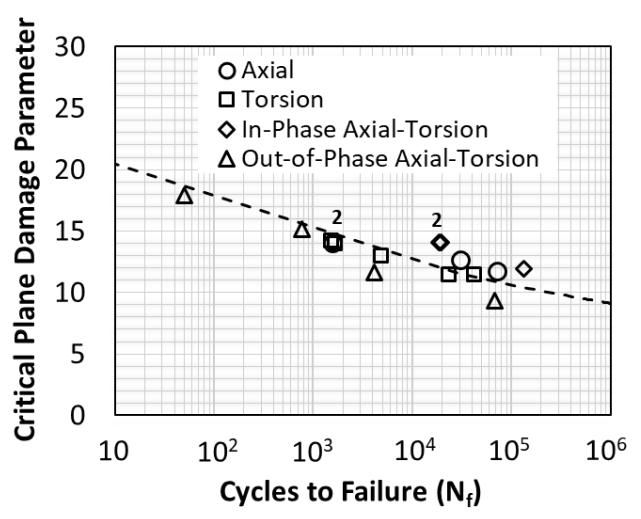

b

Fig. 3. Multiaxial fatigue data correlations for fully-reversed $(R=-1)$ loading of HDPE based on (a) von Mises stress criterion and (b) critical plane damage criterion.

\subsection{Notch Effects and Prediction}

Stress concentration is always a concern in component design with respect to fatigue life assessment. The detrimental effect of a notch on fatigue performance of materials has traditionally been quantified through the so-called fatigue notch factor $K_{f}$ [22]. The fatigue 
notch factor can be expressed as a function of both $q$ (notch sensitivity factor) and the corresponding stress concentration factor, $K_{t}$. The value of $q$ varies between 0 (no notch sensitivity) and 1 (full notch sensitivity). Peterson [23] suggested a formula for $K_{f}$ as:

$$
K_{f}=1+\frac{K_{t}-1}{1+\frac{a}{r}}
$$

where $r$ is notch root radius and $a$ is material characteristic length. An empirical relationship between $a$ and $S_{u}$ for a range of thermoplastic materials was suggested by Mortazavian and Fatemi [24] as:

$$
a=5.67 S_{u}^{-0.375}
$$

where $S_{u}$ is in $\mathrm{MPa}$ and $a$ is in $\mathrm{mm}$. In order to construct the prediction line for notch effect, two points are used, the first point by assuming equal strength of un-notched and notched specimens at first cycle, and the second point by estimating the fully-reversed notched fatigue strength at long life (here taken as $10^{6}$ cycles) based on the un-notched fatigue strength divided by $K_{f}$. The two points are then connected linearly in a log-log plot.

A limited number of tests were conducted for notch effect on fatigue life of HDPE. Shear stress and equivalent von Mises stress are used for torsional and in-phase loadings, respectively. The superimposition of the un-notched and notched data are shown in Fig. 4. As can be seen, the predictions are reasonable based on this simple methodology. It should be noted that this approach is based on nominal applied stress and does not require determination of local stresses or strains.

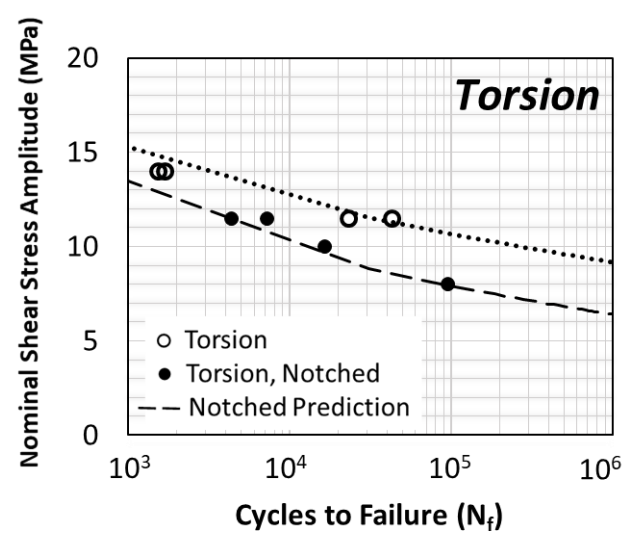

a

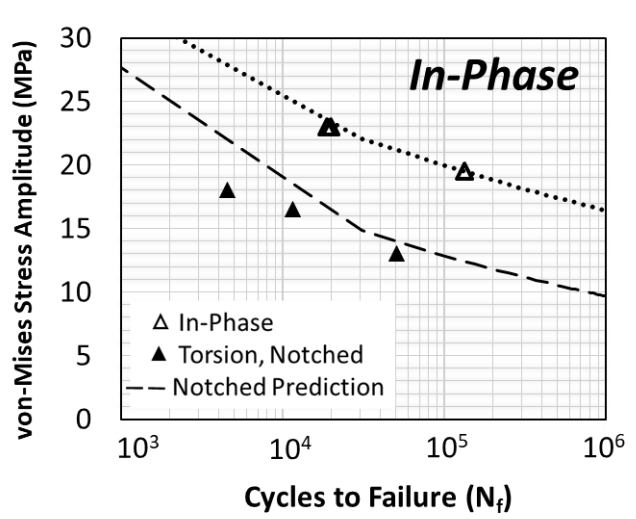

b

Fig. 4. Notch effect experimental data and modeling for fully-reversed $(R=-1)$ torsional (a) and inphase axial-torsion (b) loadings.

\section{Conclusions}

Multiaxial fatigue behavior of HDPE under axial, torsion, in-phase, and out-of-phase axialtorsion was studied by conducting fully reversed load-controlled fatigue tests on thin-walled tubular specimens. Self-heating was minimized by keeping the temperature rise below $5^{\circ} \mathrm{C}$. A limited number of tests were also performed to investigate notch effect on fatigue behavior of HDPE. Damage mechanism was studied for un-notched and notched specimens. Based on 
the observed experimental results and analysis performed, the following conclusions can be made:

1. Crack initiation mechanism in HDPE is shear which was observed for both unnotched and notched specimens under either torsion or in-phase axial-torsion loading conditions. Crack initiation orientations were all along maximum shear stress plane. For in-phase axial-torsion loading in both un-notched and notched tests, once cracks initiate, the growth orientation was along mode I crack growth direction.

2. The common von Mises criterion could correlate axial, torsion, and in-phase axialtorsion fatigue data together. However, it could not account for the effect of load non-proportionality on multiaxial fatigue data of HDPE.

3. Stress version of a critical plane based damage parameter correlated all multiaxial fatigue data well.

4. The nominal stress-based approach based on fatigue notch factor could account for notch effect on fatigue life under torsion and in-phase axial-torsion loading conditions. Fatigue life predictions for notched tests correlated well with the limited notched experimental data.

\section{References}

1. L. Tavazzi, The excellence of the plastics supply chain in relaunching manufacturing in italy and europe. The European House, Ambrosetti, (2013)

2. F. Awaja, S. Zhang, M. Tripathi, A. Nikiforov, and N. Pugno, Cracks, microcracks and fracture in polymer structures: Formation, detection, autonomic repair. Prog. in Mat. Sci., 83: pp. 536-573 (2016)

3. R. Popli and L. Mandelkern, Influence of structural and morphological factors on the mechanical properties of the polyethylenes. J. of Polym. Sci. Part B: Polymer Physics, 25: pp. 441-483 (1987)

4. Y. Ulcer, M. Cakmak, J. Miao, and C.M. Hsiung, Structural gradients developed in injection-molded syndiotactic polystyrene (sPS). J. of App. Polym. Sci., 60: pp. 669691 (1996)

5. H. Zhou and G.L. Wilkes, Orientation-dependent mechanical properties and deformation morphologies for uniaxially melt-extruded high-density polyethylene films having an initial stacked lamellar texture. J. of Mat. Sci., 33: pp. 287-303 (1998)

6. F. Addiego, A. Dahoun, C. G'Sell, J.-M. Hiver, and O. Godard, Effect of microstructure on crazing onset in polyethylene under tension. Polym. Eng. \& Sci., 49: pp. 11981205 (2009)

7. H.E.H. Meijer and L.E. Govaert, Mechanical performance of polymer systems: The relation between structure and properties. Prog. in Polym. Sci., 30: pp. 915-938 (2005)

8. A.N. Karasev, I.N. Andreyeva, N.M. Domareva, K.I. Kosmatykh, M.G. Karaseva, and N.A. Domnicheva, Relationship between the mechanical behaviour and molecular weight distribution of high density polyethylene. Polym. Sci. U.S.S.R., 12: pp. 12751288 (1970)

9. M. Hiroyuki, O. Masaki, and K. Kikuo, Effects of strain rate and relaxation rate on elastic modulus of semi-crystalline polymer. Jap. Soc. for Comp. Meth. in Eng., 7: p. $6(2008)$ 
10. S. Lampman, Characterization and Failure Analysis of Plastics, ASM International (2003)

11. B.A.G. Schrauwen, Deformation and Failure of Semi-crystalline Polymer Systems: Influence of Micro and Molecular Structure, Doctoral Thesis, Eindhoven University of Technology (2003)

12. K. Chen, G. Kang, F. Lu, and H. Jiang, Uniaxial cyclic deformation and internal heat production of ultra-high molecular weight polyethylene. J. of Polym. Reser., 22: p. 217 (2015)

13. A. Berrehili, S. Castagnet, and Y. Nadot, Multiaxial fatigue criterion for a high-density polyethylene thermoplastic. Fat. \& Frac. of Eng. Mat. \& Struct., 33: pp. 345-357 (2010)

14. ASTM E2207-15, Standard Practice for Strain-Controlled Axial-Torsional Fatigue Testing with Thin-Walled Tubular Specimens. ASTM International (2017)

15. P.E. Bretz, R.W. Hertzberg, and J.A. Manson, Mechanisms of fatigue damage and fracture in semi-crystalline polymers. Polym., 22: pp. 1272-1278 (1981)

16. J.A. Sauer and G.C. Richardson, Fatigue of polymers. Int. J. of Fract., 16: pp. 499532 (1980)

17. A. Pawlak, A. Galeski, and A. Rozanski, Cavitation during deformation of semicrystalline polymers. Prog. in Polym. Sci., 39: pp. 921-958 (2014)

18. A. Fatemi and N. Shamsaei, Multiaxial fatigue: An overview and some approximation models for life estimation. Int. J. of Fat., 33: pp. 948-958 (2011)

19. N.R. Gates, Fatigue Behavior under Multiaxial Stress States Including Notch Effects and Variable Amplitude Loading, Doctoral Thesis, University of Toledo (2016)

20. A. Fatemi and D.F. Socie, A Critical Plane Approach to Multiaxial Fatigue Damage Including Out-of-Phase Loading. Fat. \& Fract. of Eng. Mat. \& Struct., 11: pp. 149165 (1988)

21. N.R. Gates and A. Fatemi, On the consideration of normal and shear stress interaction in multiaxial fatigue damage analysis. Int. J. of Fat., 100: pp. 322-336 (2017)

22. R.I. Stephens, A. Fatemi, R.R. Stephens, and H.O. Fuchs, Metal Fatigue in Engineering, John Wiley \& Sons (2000)

23. R.E. Peterson, Stress Concentration Factors. John Wiley and Sons, New York (1974)

24. S. Mortazavian and A. Fatemi, Effects of mean stress and stress concentration on fatigue behavior of short fiber reinforced polymer composites. Fat. \& Fract. of Eng. Mat. \& Struct., 39: pp. 149-166 (2016) 